\title{
MAGNETIC PROPERTIES OF RAPIDLY OSCILLATING AP STARS
}

\author{
G. MATHYS \\ European Southern Observatory \\ Casilla 19001, Santiago 19, Chile
}

AND

S. HUBRIG

University of Potsdam

Am Neuen Palais 10, D-14469 Potsdam, Germany

\section{Introduction}

Rapidly oscillating Ap stars generally pulsate in multiple modes, characterized by different frequencies. The amplitudes of these modes may furthermore be modulated with the rotation frequency of the star. For the two roAp stars whose magnetic fields have been sufficiently studied, the maximum pulsation amplitude coincides in phase with one of the extrema of the mean longitudinal magnetic field. Two interpretations of this property have been proposed: the oblique pulsator model, according to which the pulsation modes are aligned with the magnetic axis of the star, and the spotted pulsator model, which assumes that the pulsation modes are symmetric about the rotation axis of the star, and that pulsation amplitude modulation is due to the inhomogeneity of the stellar surface (which, itself, is related to the magnetic field geometry). At present, no definite choice between these two models can be made, though the oblique pulsator model is often preferred.

Another unsolved question is that of the excitation mechanism of the pulsation. Plausible candidates seem to be the $\kappa$-mechanism restricted to the magnetic poles in the He II or Si IV ionization zones, or magnetic overstability.

In short, the presence of a magnetic field is required by all the mechanisms driving the pulsation that have been proposed as well as by both models suggested to explain the observed modulation of the amplitude of the rapid oscillations. This implies that the proper interpretation of the seismological behaviour of the roAp stars requires the knowledge of their magnetic fields. The latter is still very incomplete: here we summarize its current status.

\section{Magnetic Field Observations in roAp Stars}

Unless otherwise noted, the data summarized here are presented in greater detail in Mathys et al. (1997) and Mathys and Hubrig (1997), and references therein. The 
reader is referred to those papers for additional information.

Among the 28 roAp stars known:

- 14 definitely or very probably have a magnetic field: HD 6532, HD 19918, HD 24712, HD 80316, HD 83368, HD 101065 (Wolff and Hagen 1976), HD 119027, HD 128898, HD 134214, HD 137949, HD 150562, HD 166473, HD 176232, and HD 201601;

- of these 14, only 2 (HD 24712 and HD 83368) have been studied throughout their rotation cycle;

- magnetic fields have been sought in 4 additional stars, but only upper limits could be obtained: HD 193756, HD 203932, HD 217522, and HD 218495;

- for 10 stars, there have been no magnetic field determinations so far. These stars are HD 9289, HD 12932, HD 42659, HD 60435, HD 84041, HD 86181, HD 161459, HD 185256, HD 190290, and HD 196470.

\section{Conclusion}

In spite of the importance of magnetic fields for the proper understanding of the pulsational properties of the roAp stars, these fields have only been scarcely studied until now. An additional observational effort is necessary. Accordingly, we have undertaken a program to study more systematically the magnetic fields of the roAp stars.

\section{References}

Mathys, G. and Hubrig, S.: 1997, Astron. Astrophys. Suppl. Series 124, 475

Mathys, G., Hubrig, S., Landstreet, J.D., Lanz, T. and Manfroid, J.: 1997, Astron. Astrophys. Suppl. Series 123, 353

Wolff, S.C. and Hagen, W.: 1976, Publ. Astron. Soc. Pacific 88, 119 